\title{
Impoliteness (Hate Speech/Incivility)
}

\section{AUTHOR}

Katharina Esau

\section{KEYWORDS}

impoliteness, rudeness, politeness, respect, disrespect, insulting language, vulgarity, aggressive language, incivility, civility

\section{BRIEF DESCRIPTION}

The variable impoliteness is an indicator used to describe violations of communication norms. These norms can be social norms established within a society, a culture or parts of a society (e.g. a social class, milieu or group). In this sense impoliteness is associated with, among other things, aggressive, offensive or derogatory communication expressed directly or indirectly to other individuals or parties. More specifically name calling, vulgar expressions or aspersions are classified as examples of impolite statements (e.g. Papacharissi, 2004; Seely, 2017). While some scholars distinguish between impoliteness and incivility and argue that impoliteness is more spontaneous, unintentional and more frequently regretted than incivility (e.g. Papacharissi, 2004; Rowe, 2015), other scholars include impoliteness into the concept of incivility and argue that the two concepts have no clear boundaries (Coe, Kenski, \& Rains, 2014; e.g. Seely, 2017). In many studies a message is classified as impolite if the message contains at least one instance of impoliteness (e.g. a swear word). The direction of an impolite statement is coded as ,interperso-

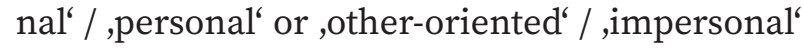
or sometimes also as ,neutral', meaning it is not directed at any group or individual

FIELD OF APPLICATION/THEORETICAL FOUNDATION Impoliteness is a broader concept of violati- ons of norms in communication that, in digital communication research, is often referred to in studies on incivility. Politeness can be related to theories on social norms of communication and conversation, for example conversational-maxims (Grice, 1975), face-saving concepts (Brown \& Levinson, 1987; Goffman, 1989) or conversational-contract theories (Fraser, 1990).

\section{REFERENCES/COMBINATION WITH OTHER METHODS OF DATA COLLECTION}

Impoliteness is examined through content analysis and is sometimes combined with comparative designs (e.g., Rowe, 2015) or experimental designs (Muddiman, 2017; Oz, Zheng, \& Chen, 2017). In addition, content analyses can be accompanied by interviews or surveys, for example to validate the results of the content analysis (Erjavec \& Kovačič, 2012).

\section{EXAMPLE STUDIES}

Research question/research interest: Previous studies have been interested in the extent, levels and direction of impoliteness in online communication (e.g. in one specific online discussion, in discussions on a specific topic, in discussions on a specific platform or on different platforms comparatively).

Object of analysis: Previous studies have investigated impoliteness in user comments on political newsgroups, news websites, social media platforms (e.g. Twitter, Facebook), political blogs, science blogs or online consultation platforms.

Timeframe of analysis: Content analysis studies investigate impoliteness in user comments focusing on periods between 2 months and 1 year (Coe et al., 2014; Rowe, 2015; Seely, 2017). It is common to use constructed weeks. 
Level of analysis: Most manual content analysis studies measure impoliteness on the level of a message, for example on the level of user comments. On a higher level of analysis, the level of impoliteness for a whole discussion thread or online platform could be measured or estimated. On a lower level of analysis impoliteness can be measured on the level of utterances, sentences or words which are the preferred levels of analysis in automated content analyses.

Table 1. Previous manual content analysis studies and measures of impoliteness.

\section{Example study Construct Dimensions/variables Explanation/example}

Reliability

\begin{tabular}{|c|c|c|c|c|}
\hline \multirow[t]{10}{*}{$\begin{array}{l}\text { Papacharissi } \\
(2004)\end{array}$} & \multirow{10}{*}{$\begin{array}{l}\text { impolite- } \\
\text { ness } \\
\text { (separate } \\
\text { from incivi- } \\
\text { lity) }\end{array}$} & name-calling & $\begin{array}{l}\text { e.g. "weirdo", "traitor", } \\
\text { "crackpot" }\end{array}$ & $\operatorname{Ir}=.91$ \\
\hline & & aspersion & e.g. "reckless", "irrational", & $\operatorname{Ir}=.91$ \\
\hline & & synonyms for liar & e.g. "hoax", "farce" & N/A \\
\hline & & hyperboles & e.g. "outrageous”, “heinous” & N/A \\
\hline & & non-cooperation & - & N/A \\
\hline & & pejorative speak & - & N/A \\
\hline & & vulgarity & e.g. "shit", "damn", "hell” & $\operatorname{Ir}=.89$ \\
\hline & & sarcasm & - & $\mathrm{N} / \mathrm{A}$ \\
\hline & & all-capital letters & used online to reflect shou- & N/A \\
\hline & & impoliteness & & $\operatorname{Ir}=.90$ \\
\hline $\begin{array}{l}\text { Coe et al. } \\
(2014)\end{array}$ & $\begin{array}{l}\text { impolite- } \\
\text { ness } \\
\text { (included }\end{array}$ & name-calling & $\begin{array}{l}\text { mean-spirited or dispara- } \\
\text { ging words directed at a } \\
\text { person or group of people }\end{array}$ & $\mathrm{K}-\mathrm{a}=.67$ \\
\hline & & aspersion & $\begin{array}{l}\text { mean-spirited or dispara- } \\
\text { ging words directed at an } \\
\text { idea, plan, policy, or beha- } \\
\text { vior }\end{array}$ & $K-a=.61$ \\
\hline
\end{tabular}




\begin{tabular}{|c|c|c|c|c|}
\hline & & reference to lying & $\begin{array}{l}\text { stating or implying that an } \\
\text { idea, plan, or policy was } \\
\text { disingenuous }\end{array}$ & $\mathrm{K}-\mathrm{a}=.73$ \\
\hline & & vulgarity & $\begin{array}{l}\text { using profanity or language } \\
\text { that would not be conside- } \\
\text { red proper (e.g., "pissed", } \\
\text { "screw") in professional } \\
\text { discourse }\end{array}$ & $K-\alpha=.91$ \\
\hline & & $\begin{array}{l}\text { pejorative for } \\
\text { speech }\end{array}$ & $\begin{array}{l}\text { disparaging remark about } \\
\text { the way in which a person } \\
\text { communicates }\end{array}$ & $K-a=.74$ \\
\hline & & $\begin{array}{l}\text { impoliteness/inci- } \\
\text { vility }\end{array}$ & & $\mathrm{K}-\mathrm{a}=.73$ \\
\hline \multirow{5}{*}{ Rowe (2015) } & \multirow{5}{*}{$\begin{array}{l}\text { impolite- } \\
\text { ness } \\
\text { (separate } \\
\text { from incivi- } \\
\text { lity) }\end{array}$} & name-calling & e.g., "gun-nut”, “idiot”, “fool” & $\kappa=.82$ \\
\hline & & aspersion & $\begin{array}{l}\text { comments containing an } \\
\text { attack on the reputation or } \\
\text { integrity of someone or } \\
\text { something }\end{array}$ & $\kappa=.72$ \\
\hline & & lying & $\begin{array}{l}\text { comments implying disinge- } \\
\text { nuousness }\end{array}$ & $\mathrm{N} / \mathrm{A}$ \\
\hline & & vulgarity & $\begin{array}{l}\text { e.g., "crap", "shit”, any swe- } \\
\text { ar-words/cursing, sexual } \\
\text { innuendo }\end{array}$ & $\kappa=1$ \\
\hline & & pejorative & $\begin{array}{l}\text { comments containing lan- } \\
\text { guage which disparage the } \\
\text { manner in which someone } \\
\text { communicates (e.g., blather, } \\
\text { crying, moaning) }\end{array}$ & $\kappa=1$ \\
\hline
\end{tabular}




\begin{tabular}{|c|c|c|c|c|}
\hline & & hyperbole & $\begin{array}{l}\text { a massive overstatement } \\
\text { (e.g., makes pulling teeth } \\
\text { with pliers look easy) }\end{array}$ & $\kappa=.75$ \\
\hline & & non-cooperation & $\begin{array}{l}\text { a situation in a discussion in } \\
\text { terms of a stalemate }\end{array}$ & $\kappa=.66$ \\
\hline & & sarcasm & - & $\kappa=.71$ \\
\hline & & other impoliteness & $\begin{array}{l}\text { any other type of impolite- } \\
\text { ness }\end{array}$ & $\kappa=.72$ \\
\hline & & impoliteness & & $\kappa=.78$ \\
\hline \multirow[t]{5}{*}{ Seely (2017) } & \multirow{5}{*}{$\begin{array}{l}\text { impolite- } \\
\text { ness } \\
\text { (included } \\
\text { in incivility) }\end{array}$} & insulting language & $\begin{array}{l}\text { name calling and other de- } \\
\text { rogatory remarks often seen } \\
\text { in pejorative speech and }\end{array}$ & $K-a=.84$ \\
\hline & & stereotyping of poli- & e.g. "typical lying lefties" & $K-a=.88$ \\
\hline & & $\begin{array}{l}\text { stereotyping using } \\
\text { "isms"/discrimina- } \\
\text { tory language }\end{array}$ & $\begin{array}{l}\text { e.g. "if we don't get rid of } \\
\text { idiotic Muslim theologies, } \\
\text { we will have growing prob- }\end{array}$ & $K-a=1$ \\
\hline & & $\begin{array}{l}\text { other stereotyping } \\
\text { language }\end{array}$ & $\begin{array}{l}\text { e.g. “GENERALS LIKE TO } \\
\text { HAVE A MALE SOLDIER ON } \\
\text { THEIR LAP AT ALL TIMES.” }\end{array}$ & $K-a=.78$ \\
\hline & & sarcasm & $\begin{array}{l}\text { e.g. "betrayed again by the } \\
\text { Repub leadership ... what a } \\
\text { shock" }\end{array}$ & $\mathrm{K}-\mathrm{a}=.79$ \\
\hline
\end{tabular}




\begin{tabular}{lll} 
accusations of lying & e.g. "typical lying lefties" & K- $\alpha=.80$ \\
\hline shouting & $\begin{array}{l}\text { excessive capitalization } \\
\text { and/or exclamation points }\end{array}$ & K- $\alpha=.83$
\end{tabular}

impoliteness/inci-

$\mathrm{K}-\mathrm{a}=.81$

vility

Note: Previous studies used different inter-coder reliability statistics: $\mathrm{Ir}=$ reliability index by Perreault and Leigh (1989); K- $\alpha=$ Krippendorff's- $\alpha$; $\kappa$ = Cohen's Kappa

Codebook used in the study Rowe (2015) is available under: https://www.tandfonline.com/doi/fu 11/10.1080/1369118X.2014.940365

\section{REFERENCES}

Brown, P., \& Levinson, S. C. (1987). Politeness: Some universals in language usage. Cambridge: Cambridge University Press.

Coe, K., Kenski, K., \& Rains, S. A. (2014). Online and Uncivil? Patterns and Determinants of Incivility in Newspaper Website Comments. Journal of Communication, 64(4), 658-679. https://doi.org/10.1111/jcom.12104

Erjavec, K., \& Kovačič, M. P. (2012). "You Don't Understand, This is a New War! ” Analysis of Hate Speech in News Web Sites' Comments. Mass Communication and Society, 15(6), 899-920. https:// doi.org/10.1080/15205436.2011.619679

Fraser, B. (1990). Perspectives on politeness. Journal of Pragmatics, 14(2), 219-236. https:// doi.org/10.1016/0378-2166(90)90081-n

Goffman, E. (1989). Interaction ritual: Essays on face-to-face behavior. New York: Pantheon Books.

Grice, P. H. (1975). Logic and conversation. In P. Cole (Ed.), Syntax and Semantics: Speech acts (pp. 41-58). New York: Academic Press.

Muddiman, A. (2017). : Personal and public levels of political incivility. International Journal of Communication, 11, 3182-3202.
Oz, M., Zheng, P., \& Chen, G. M. (2017). Twitter versus Facebook: Comparing incivility, impoliteness, and deliberative attributes. New Media \& Society, 20(9), 3400-3419. https://doi.org/10.1177/1461444817749516

Papacharissi, Z. (2004). Democracy online: Civility, politeness, and the democratic potential of online political discussion groups. New Media \& Society, 6(2), 259-283. https://doi.org/10.1177/1461444804041444

Rowe, I. (2015). Civility 2.0: A comparative analysis of incivility in online political discussion. Information, Communication \& Society, 18(2), 121-138. https:// doi.org/10.1080/1369118X.2014.940365

Seely, N. (2017). Virtual Vitriol: A Comparative Analysis of Incivility Within Political News Discussion Forums. Electronic News, 12(1), 42-61. https:// doi.org/10.1177/1931243117739060 